\title{
Synchronous leiomyosarcoma and fibroma in a single ovary: A case report and review of the literature
}

\author{
MENG HE $^{1 *}$, YAN-JIE DENG ${ }^{2 *}$, DAN-YI ZHAO ${ }^{1}$, YANG ZHANG $^{1}$ and TAO WU ${ }^{1}$ \\ ${ }^{1}$ Department of Oncology, Second Affiliated Hospital of Dalian Medical University, Dalian, Liaoning 116023; \\ ${ }^{2}$ Department of Gynecology, Dalian Women and Children's Health Hospital, Dalian, Liaoning 116033, P.R. China
}

Received December 1, 2014; Accepted December 15, 2015

DOI: $10.3892 / \mathrm{ol} .2016 .4241$

\begin{abstract}
Primary ovarian leiomyosarcoma (POLMS) is a rare disease. To the best of our knowledge, only 72 cases, including the present case, have been reported in the English literature, while synchronous POLMS and fibroma in a single ovary have not previously been reported at all. In the present study, a 46-year-old premenopausal woman was diagnosed with a mass in the left ovary in 2005. A total of 5 years after the diagnosis of this mass, the patient was admitted to hospital exhibiting lower abdominal pain, and two masses were observed in the left ovary. An exploratory laparoscopy was performed. Frozen section analysis led to a diagnosis of fibroma. Furthermore, the observed second mass was hypothesized to be a malignant form of the original fibroma. A hysterectomy and bilateral salpingo-oophorectomy were performed. Pathological reports following surgery revealed concurrent stage Ic POLMS and fibroma in the left ovary. A total of 13 months after the initial surgery, recurrent leiomyosarcoma was detected. Although the patient underwent multiple cytoreductive surgeries and chemotherapy cycles, as well as interstitial brachytherapy and conventional therapy, a poor state of health ensued. Due to the rarity of POLMS, particularly in combination with ovarian fibroma, the current report presents a detailed overview of the literature and discusses a number of histogenetic and clinical issues.
\end{abstract}

Correspondence to: Mr. Yang Zhang or Mr. Tao Wu, Department of Oncology, Second Affiliated Hospital of Dalian Medical University, 465 Zhongshan Road, Dalian, Liaoning 116023, P.R. China

E-mail: zydl@medmail.com.cn

E-mail: wuyihong97@163.com

${ }^{*}$ Contributed equally

Abbreviations: POLMS, primary ovarian leiomyosarcoma; $\mathrm{RI}$, resistance index; PR, progesterone receptor; ER, estrogen receptor; $\mathrm{PET/CT}$, positron emission tomography/computed tomography

Key words: ovary, leiomyosarcoma, fibroma, synchronous tumors, histogenesis

\section{Introduction}

Mesenchymal tumors are uncommon in the ovary, occurring more frequently in other regions of the body (1-3). Primary ovarian leiomyosarcoma (POLMS) and fibroma are mesenchymal tumors. Fibroma represents 3-5\% of all ovarian tumors, and its histogenesis remains to be elucidated (4). The majority of patients exhibiting ovarian fibroma are asymptomatic due to the tumor's small size (4). However, patients may experience abdominal pain, abdominal enlargement or urinary symptoms. Furthermore, ascites is a common associated finding and may be observed in half of all cases with fibroma measuring $>5 \mathrm{~cm}$ in diameter (4). When accompanied by ascites and pleural effusion, ovarian fibroma develops into Meigs' syndrome, which occurs in 1-3\% of cases. To the best of our knowledge, only 72 examples of POLMS (including the present case) have been reported (1,5-29). When leiomyosarcoma is encountered in the ovary, it may be difficult to diagnose (4); its treatment and etiology remain complicated and controversial. In addition, the prognosis of POLMS is unfavorable and $>50 \%$ of patients succumb to the disease following a mean time of 24 months (1).

To the best of our knowledge, the current report presents the first case of concurrent POLMS and fibroma in a single ovary in a premenopausal woman, and highlights the association between malignant and benign ovarian tumors. A detailed literature review concerning POLMS and fibroma is followed by the discussion of several associated histogenetic and clinical issues. Written informed consent was obtained from the patient's family for the publication of this study.

\section{Case report}

Patient and diagnosis. The patient was a 46-year-old premenopausal woman (gravida 1, para 1), who experienced regular menstrual cycles and had no relevant family history. The patient was diagnosed with an ovarian mass at Dalian Women and Children's Health Hospital (Dalian, China) in September 2005. The size of the mass at that time was $3 \mathrm{~cm}$ in diameter. As no symptoms associated with the abdominal mass were present, the patient was not concerned and no treatment was administered. During the following 5 years, the size of the mass did not alter and the patient was in good health. On November 5, 2010, the patient was admitted to Dalian 
Women and Children's Health Hospital (Dalian, China) with the chief complaint of lower abdominal pain, which had persisted for 4 days. Gynecological examination revealed cervical hypertrophy, and a uterus that was similar in size to that of a 40-day pregnant woman. In addition, a palpated, firm, tender mass, with poor activity and dimensions approximate to that of a duck egg, was observed to be adherent to the left posterior uterus. Color Doppler ultrasound (Medison 8000EX; Samsung Medison, Seoul, Korea) revealed two masses in the left ovary. One hybrid echoic mass was irregular in shape, and was encapsulated by a discontinuous membrane and filled with an opaque dark area of fluid, which scattered a number of thick light spots. Furthermore, color Doppler ultrasound imaging identified an abundant blood supply and indicated a vascular resistance index (RI) of 0.77 , which suggested that the mass may have been benign (17). The remaining solid mass was regular in shape, and was encapsulated by a continuous membrane and filled with a hypo-echoic area. The RI of this mass was 0.6 . These two masses measured $42 \times 42 \times 40 \mathrm{~mm}$ and $49 \times 43 \times 40 \mathrm{~mm}$ in size, respectively. The endometrial thickness was $15 \mathrm{~mm}$, which was thicker than normal. Fractional curettage revealed no abnormalities. Two uterine fibroids were detected, one measuring $21 \times 21 \times 19 \mathrm{~mm}$ located on the anterior wall of the uterus, and the other 20x18x15 mm on the posterior wall.

Laboratory test results were all normal and were as follows: Carcinoembryonic antigen, $1.98 \mathrm{ng} / \mathrm{ml}$ (normal range, 0-3.4 ng/ml), $\alpha$-fetoprotein, $<0.5 \mathrm{ng} / \mathrm{ml}$ (normal range, $0-5.8 \mathrm{ng} / \mathrm{ml}$ ); and cancer antigen $125,27.73 \mathrm{U} / \mathrm{ml}$ (normal range, $0-35 \mathrm{U} / \mathrm{ml}$ ).

On November 10, 2010, an exploratory laparoscopy was performed. An enlarged left ovary was observed, with a prominent hemorrhagic smooth mass on the surface (Fig. 1A). The mass measured $5 \mathrm{~cm}$ in diameter and exhibited no adherence to the surrounding tissue. In addition, the right ovary was atrophied. Gross examination of the excised left ovarian mass revealed a phyllodes tumor, which consisted of two regions, measuring up to $75 \times 45 \times 40 \mathrm{~mm}$. The first half of the tumor (region I) was fragile and pale inside, and exhibited small hemorrhagic areas and a fine texture. The remaining half (region II), however, demonstrated a pale yellow interior, and was resilient and firm. These two regions were partially connected to each other by an encapsulated membrane. According to frozen pathology, the phyllodes tumor was composed of spindle cells with various shapes. Region II with regular shaped cells was confirmed to be a fibroma (30). Region I exhibited a number of deformed cells with nuclear fission concentrated in certain zones, which was suspected to be malignant transformation of the fibroma. As the patient's family members refused to allow a staging laparotomy to be performed, a hysterectomy and bilateral salpingo-oophorectomy were conducted. The pelvis and greater omentum appeared grossly normal and no ascites was present. The patient underwent an uneventful post-operative course.

Pathology. Tumor tissues were sent to the Department of Pathology, Dalian Women and Children's Health Hospital (Dalian, China) for sectioning. Microscopic examination of region I of the tumor revealed a number of infarct-type necrotic and hemorrhagic areas (Fig. 1B), with pleomorphic and fusiform cells (Fig. 1C). Furthermore, the tumor exhibited areas of hypercellular and nuclear pleomorphism, with up to 15 mitotic figures (MF) per 10 high-power fields (HPF). In a number of the sections, medium-sized and smaller blood vessels were encountered with thin muscle coats, and a total absence of adventitial layers (Fig. 1D). As blood vessel walls exhibit intense immunostaining for cluster of differentiation (CD)34, anti-CD34 antibody was used for demonstrating blood vessels absence of adventitial layers. Microscopic examination of region II of the tumor revealed short spindle-shaped cells, with narrow or ovoid spindle-shaped nuclei. The cells formed bundles that were frequently intersected by hyalinized tissue. Mitotic activity was absent (Fig. 1E).

Tumor tissues were sent to the Department of Pathology, Dalian Women and Children's Health Hospital (Dalian, China), for immunohistochemical staining. Immunohistochemical staining of region II of the tumor revealed smooth muscle actin negativity, while region I exhibited positivity for vimentin, SMA (Fig. 1F), desmin, caldesmon and p53, and negative results for inhibin, Ki-67, progesterone receptor (PR) and estrogen receptor (ER). The final pathology provided a diagnosis of concurrent fibroma and stage Ic leiomyosarcoma in a single ovary (FIGO staging) (31), which was further confirmed by Peking Union Medical College Hospital (Beijing, China).

Post-operative positron emission tomography/computed tomography (PET/CT) observed no additional tumor foci remaining in the patient's body, indicating that the leiomyosarcoma was POLMS. The patient underwent a close follow-up subsequent to surgery, and refused chemotherapy. A total of 13 months after the initial surgery, PET/CT revealed multiple nodules in the pelvis. Cytoreductive surgery was performed at Peking Union Medical College Hospital so that no residual disease remained. The post-operative pathological diagnosis confirmed recurrent POLMS. Subsequently, 4 cycles of adjuvant chemotherapy were performed. The first cycle was docetaxel plus gemcitabine (90 mg docetaxel on day 1 and $1 \mathrm{~g}$ gemcitabine on day 2; 21 days; one cycle), and the following 3 cycles consisted of gemcitabine alone due to the patient demonstrating intolerance to docetaxel (total duration of 4 cycles was 3 months). A total of 11 months after the second surgery, PET/CT confirmed a relapse of nodules in the pulmonary organs, a $13-\mathrm{cm}$ mass in the left kidney and a high signal intensity in segment 4 of the liver. Cytoreductive surgery was conducted for a second time, with the pulmonary nodules remaining. Immunohistochemical staining following the surgery revealed total negativity for CD117, epidermal growth factor receptor and human epidermal growth factor receptor-2.

Genetic testing was performed at the Cancer Institute and Hospital, Chinese Academy of Medical Sciences (Beijing, China) using second-generation sequencing technology. The result additionally indicated total negativity for vascular endothelial growth factor (VEGF) receptor, B-raf V600E, phosphatidylinositol-4,5-bisphosphate 3-kinase, catalytic subunit $\alpha$ codon 545 and echinoderm microtubule-associated protein-like 4-anaplastic lymphoma kinase confusing gene. Thus, no suitable targeted therapy could be administered, and the patient's disease gradually progressed. A total of 7 months after the third surgery, the patient was administered 4 cycles of adjuvant chemotherapy with epirubicin, ifosfamide and bevacizumab (50 mg epirubicin on days 1 and 2, $2 \mathrm{mg}$ ifosfamide 

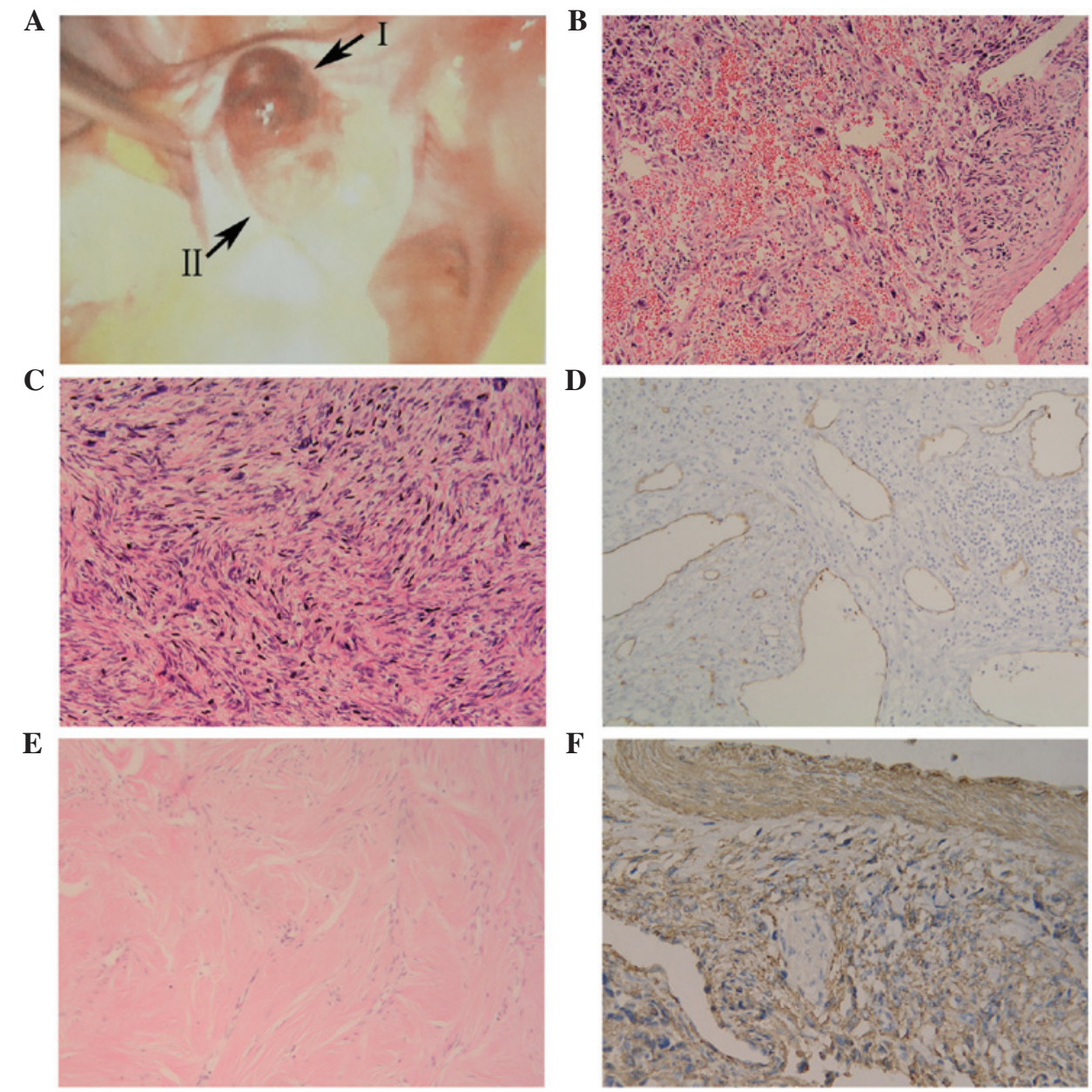

$\mathbf{E}$

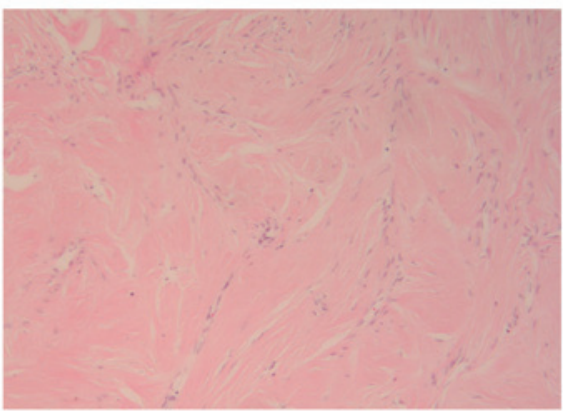

Figure 1. Imaging studies of the ovarian mass. (A) Exploratory laparoscopy revealing a mass in the enlarged left ovary, composed of two regions (regions I and II). Microscopic examination of region I revealed (B) focal hemorrhage and necrosis (hematoxylin and eosin staining; magnification, x100) (C) with pleomorphic and fusiform cells (hematoxylin and eosin staining; magnification, x200). (D) Blood vessels demonstrating an absence of adventitial layers (blood vessel wall exhibiting intense immunostaining for cluster of differentiation 34; magnification, x100) in a section of region I. (E) Microscopic examination of region II (hematoxylin and eosin staining; magnification, x100). (F) Immunohistochemical staining of region I was SMA (ovarian leiomyosarcoma demonstrating intense immunostaining for SMA; magnification, x200). SMA, smooth muscle actin.

on days 1-5 and $6 \mathrm{mg}$ bevacizumab on days 1 and 15 ; total duration of 4 cycles was 3 months); however, during this time the tumor progressed rapidly. Several months later, the tumor demonstrated extensive invasion. The patient underwent interstitial brachytherapy and interventional therapy; however, these treatment attempts proved ineffective. The patient received supportive care until she succumbed in January 2015.

\section{Discussion}

POLMS and fibroma occupy a small number of all ovarian neoplasms (32). To the best of our knowledge, concurrent POLMS and fibroma in a single ovary has not been previously reported. However, a number of cases have referred to an association between POLMS and fibroma. Seracchioli et al (5) reported the case of a 20-year-old woman exhibiting POLMS, who had suffered from nevoid basal cell carcinoma syndrome at $\sim 1$ year old. A total of 4 years prior to the patient's POLMS excision, a number of fibromas containing microcalcification were identified in the left ovary. A total of 3 months after the POLMS excision, multiple small fibromas were identified in the patient's remnant left ovary. In this case, POLMS and fibroma were reported to exist in one ovary asynchronously (5). Lerwill et al (1) reported a case of bilateral ovarian tumors with leiomyosarcoma in one ovary and fibroma in the other. The 82-year-old female patient succumbed to disease 10 months after the diagnosis. Seinera et al (33) reported the case of a 25-year-old woman who exhibited multiple nodules in the bilateral ovaries. Fibrous connective tissues were observable in the larger tumor. Immunohistochemical analysis confirmed ovarian leiomyoma. Additionally, Trabelsi et al (18) reported a case of myxoid ovarian leiomyosarcoma, which was dissociated by fibrohyalinized tissue in certain areas. Consequently, to the best of our knowledge, the present case is the first case of concurrent POLMS and fibroma. In order to investigate the association between these two ovarian tumors, an overview of the tumors is followed by a detailed discussion of the histogenesis.

Fibroma represents $3-5 \%$ of all ovarian tumors, and primarily occurs at peri- and postmenopause (4). Fibromas are benign tumors, and the median age of occurrence is reported to be $\sim 52$ years (4). Although certain patients are admitted to hospital with abdominal pain, abdominal enlargement or urinary symptoms, ovarian fibroma is typically asymptomatic. 
When accompanied by ascites and pleural effusion, ovarian fibroma develops into Meigs' syndrome (4), which is observed in 1-3\% of all ovarian fibromas. Meigs' syndrome resolves following resection of the ovarian fibroma. The histogenesis of ovarian fibroma remains to be elucidated, however, mesenchymal cells of the ovarian stroma, fibrosed thecoma, Brenner tumor, ovarian cortex, the walls of the blood and lymphatic vessels are all potential origins $(4,34)$.

POLMS has been reported in patients aged between 12 and 84 years (1,5-29). Among the 72 reported cases, $69 \%$ of patients were $>50$ years old. Although POLMS is typically regarded as being associated with postmenopausal women $(6,8,16)$, several of the reported cases occurred during the pediatric period $(6,12)$. A total of 4 cases occurred in patients $<20$ years, 3 of whom had suffered from medulloblastoma at the age of 1 year. The details of the fourth patient are unknown $(1,5,6,12)$. The average age of occurrence for all 72 patients was 54.9 years.

Ovarian leiomyosarcoma typically has no significant symptoms (14). However, a number of patients were referred to the hospital with abdominal pain or fullness, a few exhibited constipation and 1 patient exhibited post-menopausal bleeding (27). Based on all 72 reported cases of POLMS, it was not possible to conclude any specific clinical manifestations.

The histogenesis of ovarian leiomyosarcoma remains to be elucidated, and a number of hypothetical locations have been proposed, including a totipotent ovarian mesenchyme (35), smooth muscle fibers of ovarian ligaments (15), the vascular wall $(13,14)$, and the medulla and hilus $(12)$. Ovarian leiomyosarcoma may additionally have an origin in ovarian teratoma (35), preexisting serous cystadenoma and papillary serous cystadenocarcinoma $(18,35,36)$. It is possible, however, that not all leiomyosarcomas of the ovary originate in an identical manner or that a single leiomyosarcoma may possess a number of origins.

Although the etiology of synchronous POLMS and fibroma remains to be elucidated, the present study proposed that their occurrence is not coincidental. As ovarian fibroma and leiomyosarcoma are ovarian non-specific supporting mesenchymal tissue tumors, their development may involve common carcinogenic agents. Additionally, in a number of the pathological sections investigated in the present study, medium-sized and smaller blood vessels were observed, with thin muscle coats and a total absence of adventitial layers. This suggested an identical origin for ovarian fibroma and POLMS from smooth muscle fibers of the vascular wall. Wellmann (13) published similar findings. Following the third surgery, the present patient accepted 3 cycles of bevacizumab, a recombinant humanized monoclonal antibody that blocks angiogenesis by inhibiting VEGF-A. However, no improvement was demonstrated. The genetic tests, which demonstrated VEGF negativity, subsequently explained the ineffectiveness of bevacizumab treatment.

In addition to the aforementioned discussion points, compared with the former 71 cases, the present case merited attention in additional aspects. Clinically, POLMS requires differentiation with alternative ovarian sarcomas, tumors in other areas of the body and metastasis to the ovary $(1,5,8,37)$. Under normal conditions, ultrasonic inspection is the preferred primary diagnostic method due to its convenience.
Based on the theory of ultrasonics, a high impedance value ( $\mathrm{RI}>0.6)$ reveals that a mass is likely to be benign, while a low impedance value $(\mathrm{RI}<0.4)$ reveals that a mass is likely to be malignant (17). However, as the echo texture of solid pelvic tumors is typically non-specific, these RI criteria are not completely accurate for the diagnosis of ovarian tumors. In the present case, prior to surgery, color Doppler sonography identified a mass with malignant features, while the RI value (0.77) indicated a benign result. Additionally, Kuscu et al (17) noted that the RI of a previous ovarian leiomyosarcoma case was 0.54 , which was an intermediate value lacking diagnostic weight. Thus, clinicians should be aware that the RI value is not necessarily able to differentiate between benign and malignant ovarian masses. By contrast, pathological diagnosis has played a critical role in all reported cases of ovarian leiomyosarcoma. The cell morphology may visually exhibit the characteristics of malignant tumors. Lerwill et al (1) additionally proposed that $\geq 5 \mathrm{MF}$ per $10 \mathrm{HPF}$ in the presence of significant atypia is a guiding principle for a diagnosis of leiomyosarcoma, even in the absence of tumor cell necrosis $(1,6)$. Furthermore, immunohistochemical staining for ovarian leiomyosarcoma is typically positive for desmin and muscle-specific actin, and negative for cytokeratins and S100 $(1,11,36)$. Thus, cell morphology, as well as mitosis criteria and immunohistochemical staining, together establish the gold standard for the diagnosis of ovarian leiomyosarcoma.

The effect of hormones on POLMS additionally requires investigation $(1,24,27,35,38-40)$. The majority of patients exhibiting POLMS are postmenopausal, implying that this tumor may be associated with relatively low hormone levels. However, a number of previous cases have reported that POLMS occurred in an environment with high levels of hormone. Walts and Lichtenstein (35) reported the case of a patient who received Premarin (conjugated estrogens; Ayerst Laboratories, New York, NY, USA) for 5 years prior to her POLMS diagnosis. Bouie et al (24) reported the case of a patient who was undergoing ovulation induction at the time of POLMS diagnosis. Lerwill et al (1) identified that several POLMS cases in their study progressed rapidly during pregnancy. An additional study reported the case of a woman who was diagnosed with POLMS following admittance to hospital with post-menopausal bleeding (27). In the present case, the patient exhibited regular menstrual cycles when diagnosed with POLMS. The patient demonstrated an apparent right atrophic ovary and a thickened endometrium. This may indicate that the patient's left ovary was secreting hormone at an abnormally high level. In order to identify an appropriate therapy, additional immunohistochemical tests were conducted, revealing an almost totally negative outcome for PR and ER, which may suggest that this POLMS case was hormone-independent, which is different compared with the aforementioned cases. In conclusion, the effect of hormones on POLMS requires additional investigation in future studies.

Cases of POLMS are so rare that no recommended guidelines on optimal treatment have been established (8). Based on previously reported cases, surgery is the preferred treatment choice $(1,5)$. In the present case, although POLMS was removed at an early stage (Ic), as well as being relatively small in size ( $3 \mathrm{~cm}$ in diameter), and a series of elaborately designed therapies was conducted, the patient demonstrated a negative 
prognosis. It is hypothesized that this poor prognosis was due to the co-occurrence of POLMS and fibroma, which increased the uncertainty of treatment and therapeutic effects. Although no clear causal association has been established between POLMS and ovarian fibroma, such an observation may lead to a future research focus investigating synchronous benign and malignant tumors.

\section{References}

1. Lerwill MF, Sung R, Oliva E, Prat J and Young RH: Smooth muscle tumors of the ovary: A clinicopathologic study of 54 cases emphasizing prognostic criteria, histologic variants, and differential diagnosis. Am J Surg Pathol 28: 1436-1451, 2004.

2. Hamamoto Y, Shomori K, Nosaka K, Haruki T, Teshima R and Ito $\mathrm{H}$ : Prognostic significance of Minichromosome maintenance protein 7 and Geminin expression in patients with 109 soft tissue sarcomas. Oncol Lett 1: 703-709, 2010.

3. Fukuda T, Sumi T, Nakano Y, Morishita M, Nobeyama $H$, Yoshida H, Matsumoto Y, Yasui T, Honda KI and Ishiko O: Extragastrointestinal stromal tumor originating from the vulva. Oncol Lett 2: 797-799, 2011.

4. Talerman A and Path FRC: Nonspecific tumors of the ovary, including mesenchymal tumors and malignant lymphoma. In: Blaustein's Pathology of the Female Genital Tract. Kurman RJ (ed). 3rd edition. Springer, New York, NY, pp722-741, 1987.

5. Seracchioli R, Colombo FM, Bagnoli A, Trengia V and Venturoli S: Primary ovarian leiomyosarcoma as a new component in the nevoid basal cell carcinoma syndrome: A case report. Am J Obstet Gynecol 188: 1093-1095, 2003.

6. Monk BJ, Nieberg R and Berek JS: Primary leiomyosarcoma of the ovary in a perimenarchal female. Gynecol Oncol 48: 389-393, 1993.

7. Mayerhofer K, Lozanov P, Bodner K, Bodner-Adler B Mayerhofer-Gallenbacher N, Hudelist G and Czerwenka K: Immunohistochemical analysis of a primary ovarian leiomyosarcoma. Case report. Anticancer Res 23: 3433-3436, 2003.

8. Rasmussen CC, Skilling JS, Sorosky JI, Lager DJ and Buller RE: Stage IIIC ovarian leiomyosarcoma in a premenopausal woman with multiple recurrences: Prolonged survival with surgical therapy. Gynecol Oncol 66: 519-525, 1997.

9. Anderson AE, Yang X and Young RH: Epithelioid angiomyolipoma of the ovary: A case report and literature review. Int J Gynecol Pathol 21: 69-73, 2002.

10. Dixit S, Singhal S, Baboo HA, Vyas RK, Neema JP, Murthy R and Sooryanaraya U: Leiomyosarcoma of the ovary. J Postgrad Med 39: 151-153, 1993.

11. Taşkin S, Taşkin EA, Uzüm N, Ataoğlu O and Ortaç F: Primary ovarian leiomyosarcoma: A review of the clinical and immunohistochemical features of the rare tumor. Obstet Gynecol Surv 62: 480-486, 2007.

12. O'Sullivan SG, Das Narla L and Ferraro E: Primary ovarian leiomyosarcoma in an adolescent following radiation for medulloblastoma. Pediatr Radiol 28: 468-470, 1998.

13. Wellmann KF: Leiomyoma of the ovary: Report of an unusual case and review of the literature. Can Med Assoc J 85: 429-432, 1961.

14. Kobayashi Y, Murakami R, Sugizaki K, Yamamoto K, Sasaki S, Tajima N, Tajima H, Onda M and Kumazaki T: Primary leiomyoma of the ovary: A case report. Eur Radiol 8: 1444-1446, 1998.

15. Nasu $M$, Inoue $J$, Matsui $M$, Minoura $S$ and Matsubara $O$ Ovarian leiomyosarcoma: An autopsy case report. Pathol Int 50: $162-165,2000$.

16. Bodner K, Bodner-Adler B, Czerwenka K, Hudelist G, Kimberger O, Leodolter S and Mayerhofer K: Bcl-2 expression in a primary leiomyosarcoma of the ovary: A case report. Wien Klin Wochenschr 115: 191-195, 2003.

17. Kuscu E, Erkanli S, Haberal A, Ergin T, Ozdemir H and Demirhan B: Primary ovarian leiomyosarcoma: A case report. Eur J Gynaecol Oncol 26: 120-122, 2004.
18. Trabelsi A, Mutijima E, El Hossini Soua A, Gassoumi M, Bouguizane S, Mokni M, Yacoubi MT and Korbi S: Primary myxoid leiomyosarcoma of the ovary. A case report with review of the literature. Tunis Med 83: 288-291, 2005.

19. Dobbs SP, Brown LJ, Hollingworth J and Ireland D: Surgical treatment of recurrent primary ovarian leiomyosarcoma. A case report. Eur J Gynaecol Oncol 20: 172-173, 1999.

20. Arslan OS, Sumer C, Cihangiroglu G, Kanat-Pektas M and Gungor T: A rare tumor of the female genital tract: Primary ovarian leiomyosarcoma. Arch Gynecol Obstet 283 (Suppl 1): S83-S85, 2011.

21. Kurian RR, Preethi J and Remadevi A: Leiomyosarcoma of ovary - a case report. Indian J Pathol Microbiol 48: 19-20, 2005.

22. Piura B, Rabinovich A, Yanai-Inbar I, Cohen Y and Glezerman M: Primary sarcoma of the ovary: Report of five cases and review of the literature. Eur J Gynaecol Oncol 19: 257-261, 1998.

23. Inoue J, Gomibuchi $\mathrm{H}$ and Minoura S: A case of a primary ovarian leiomyosarcoma. J Obstet Gynaecol Res 26: 401-407, 2000.

24. Bouie SM, Cracchiolo B and Heller D: Epithelioid leiomyosarcoma of the ovary. Gynecol Oncol 97: 697-699, 2005.

25. Divya Ns and Srinivasamurthy V: Myxoid leiomyosarcoma of ovary - a rare case report. J Clin Diagn Res 8: FD05-FD06, 2014.

26. Zygouris D, Androutsopoulos G, Grigoriadis C, Arnogiannaki N and Terzakis E: Primary ovarian leiomyosarcoma. Eur J Gynaecol Oncol 33: 331-333, 2012.

27. Kaur J, Mishra M and Goel B: Primary ovarian leiomyosarcoma: A case report with review. Int J Reprod Contracept Obstet Gynecol 3: 258-260, 2014.

28. Khabir A, Boudawara T, Ayadi L, Kharrat M, Beyrouti I and Jlidi R: Epithelioid bilateral ovarian leiomyosarcoma: A study. Ann Pathol 23: 47-49, 2003 (In French).

29. Nogales FF, Ayala A, Ruiz-Avila I and Sirvent JJ: Myxoid leiomyosarcoma of the ovary: Analysis of three cases. Hum Pathol 22: 1268-1273, 1991.

30. Mackenzie DH: Fibroma: A dangerous diagnosis. A review of 205 cases of fibrosarcoma of soft tissues. Br J Surg 51: 607-612, 1964.

31. Petru ELuck HJ, Stuart G, Gaffney D, Millan D and Vergote I: Gynecologic Cancer Intergroup (GCIG) proposals for changes of the current FIGO staging system. European Journal of Obstetrics Gynecology \& Reproductive Biology 143: 69-74, 2009.

32. Azoury RS and Woodruff JD: Primary ovarian sarcomas: Report of 43 cases from the Emil Novak Ovarian Tumor Registry. Obstet Gynecol 37: 920-941, 1971.

33. Seinera P, Raspollini M, Privitera S, Farina C and Crana F: Bilateral ovarian leiomyoma. Acta Obstet Gynecol Scand 76: 488-489, 1997.

34. Sivanesaratnam V, Dutta R and Jayalakshmi P: Ovarian fibroma - clinical and histopathological characteristics. Int J Gynaecol Obstet 33: 243-247, 1990.

35. Walts AE and Lichtenstein I: Primary leiomyosarcoma associated with serous cystadenocarcinoma of the ovary. Gynecol Oncol 5: 81-86, 1977.

36. Lastarria D, Sachdev RK, Babury RA, Yu HM and Nuovo GJ: Immunohistochemical analysis for desmin in normal and neoplastic ovarian stromal tissue. Arch Pathol Lab Med 114: 502-505, 1990

37. Young RH and Scully RE: Sarcomas metastatic to the ovary: A report of 21 cases. Int J Gynecol Pathol 9: 231-252, 1990.

38. Brinton LA, Lamb EJ, Moghissi KS, Scoccia B, Althuis MD, Mabie JE and Westhoff CL: Ovarian cancer risk after the use of ovulation-stimulating drugs. Obstet Gynecol 103: 1194-1203, 2004.

39. Rossing MA, Daling JR, Weiss NS, Moore DE and Self SG: Ovarian tumors in a cohort of infertile women. New Engl J Med 331: 771-776, 1994.

40. Venn A, Jones P, Quinn M and Healy D: Characteristics of ovarian and uterine cancers in a cohort of in vitro fertilization patients. Gynecol Oncol 82: 64-68, 2001. 